\title{
A Novel Nontoxic Wood-Plastic Composite Cast
}

\author{
Lindfors $\mathrm{NC}^{1, *}$ and Salo $\mathrm{J}^{2}$ \\ ${ }^{1}$ Helsinki University Central Hospital, Department of Orthopedic and Hand Surgery, Helsinki University, Helsinki, \\ Finland \\ ${ }^{2}$ Helsinki University Central Hospital, Department of Orthopedics and Traumatology, Töölö Hospital and Helsinki \\ University, Helsinki, Finland
}

\begin{abstract}
Casting is one of the oldest methods in fracture treatment, yet shortcomings and problems with current materials are still clear. Here a novel composite casting material is presented. The material is thermoplastic, nontoxic, absolutely freely moldable in $3 \mathrm{D}$, and does not disturb X-ray analysis. After heating to $+62-64{ }^{\circ} \mathrm{C}$, the material can be cut with a normal pair of scissors and can be shaped to suit individual anatomy.

We also report the first clinical series of 33 wrist fractures treated with this wood-polymer composite material. The clinical results revealed that the cast is stable and highly durable, but still offers exceptional X-ray-radiolucent properties. The average casting time was $5.3 \mathrm{~min}$. Patient satisfaction was high, mainly due to the stability and the lightweight structure of the cast. The feedback from experienced orthopedic technicians was excellent.
\end{abstract}

Keywords: Cast, splinting, immobilization, fracture, wood, orthopedic equipment, orthopedic fixation devices.

\section{INTRODUCTION}

Fractured limbs have been treated with splints and casts for hundreds of years. Ancient Egyptians used wooden splints made of bark wrapped in linen. Starch-reinforced splints were used for the first time by Louis Seutin at Waterloo and William Eton introduced a Turkish style of wrapping plaster of Paris in London [1, 2]. Nikolay Pirogov, a Russian surgeon, introduced plaster casts in the Crimean War in 1854. In the same war, Antonius Mathysen, a Dutch army surgeon, also used cotton bandages filled with plaster of Paris for immobilization of extremities in injured soldiers [3]. During succeeding years the material, named for the gypsum mined in Paris, underwent several improvements. Binder ingredients such as starches, gums, and resins were added to improve adherence properties, and later on other additives for altering physical properties [4]. Although plaster of Paris was commonly used during the first half of the 1900s, some shortcomings were recognized. The splints were heavy and cumbersome, the water resistance was low, and they easily cracked, especially during weight-bearing conditions [5].

In the 1950s, fiberglass tape bandages were introduced for treatment of orthopedic injuries. The advantages of fiberglass over plaster of Paris, included improved mechanical properties, increased strength and rigidity, and reduced weight and cast cracking [6]. Despite these advantages and the comfort ability, there are still some unresolved issues. Fiberglass has toxic or harmful components, such as cyanates, and during application skin contact may cause

*Address correspondence to this author at the Töölö Hospital, Topeliuksenkatu 5, 00260 Helsinki, Finland; Tel: +358 50 4270845;

Fax: +358947187531; E-mail: nina.c.lindfors@hus.fi itching, redness, and dryness, necessitating the use of specific protection gloves when using fiberglass materials.

Animal studies have shown that skin exposure to chemical allergens can also elicit asthmatic responses [7]. Occupational asthma after exposure to casts containing methylene diphenyl diisocyanate has been reported [8]. Recently, workrelated asthma was diagnosed in two persons in Finland, due to long-term exposure to fiberglass casts and subsequent cyanate-vaporization [9]. Some casts can also be classified as special waste.

Currently used splinting and casting materials interfere with X-ray diagnostics. A plaster of Paris cast has a high mineral content that results in a thick radiopaque layer observed in X-rays. Fiberglass and currently used polymerbased materials have supporting nets, often seen on X-rays to cover the trabecular bone. These layers may interfere with accurate $\mathrm{X}$-ray diagnostics.

A novel nontoxic, wood-composite casting material for fracture treatment was recently introduced by Onbone $\mathrm{Oy}$, Espoo, Finland. The cast $\left(\right.$ Woodcast $^{\circledR}$ ) is an ecologically friendly, biodegradable, wood-plastic composite cast, with absolutely free three-dimensional (3D) molding properties and extreme strength. The material properties and an evaluation of how the cast fulfills the demands for a casting material are presented. We also report a series of 33 patients with distal radius fractures treated with this cast.

The study was conducted in accordance with the ethics principle originating in the latest version of the Declaration of Helsinki, applicable regulatory requirements, including the standards of the International Organization, and Finnish law and regulations. The study protocol was approved by the Ethics Committee of the Helsinki University Central Hospital (HUCH) and authorized by the Operative Unit of $\mathrm{HUCH}$. 


\section{MATERIALS AND METHODOLOGY}

\section{The Wood-Plastic Composite Material}

The casting material is a composite of a polymer and a woody material. The wood is derived from granular wood particles and the polymer is a thermoplastic polymer mixture selected from groups of nontoxic biodegradable polymers. The composite cast, Woodcast ${ }^{\circledR}$, gained European approval in 2010 and is marketed by Onbone Oy, Finland. The material used in the patient study was provided in the form of a thoroughly uniform (width $\mathrm{x}$ length $\mathrm{x}$ thickness of $10 \mathrm{x} 40 \mathrm{x} 0.4$ $\mathrm{cm}$ ) homogenous plate.

\section{Mechanical Strength, Adhesion Properties, and Skin Irritation Test of the Wood-Composite Material}

The composite casting material is strongly self-adhesive when heated. A self-adhesion wedge test was performed according to the modified standard SFS-EN 14509 (Standard Test Method for Adhesive-Bonded Surface Durability of Aluminum). The wedge test is one of the few test methods that allows testing of the quality of bonds under the influence of mechanical loading. To carry out the test, two composite plates of a prescribed thickness are bonded to each other. A wedge is driven carefully into the bond and marked as a starting point. The bond that is under stress from the wedge is forced further apart. The force required for bond breaking is measured. After the pressing force has reached equilibrium, the test is ended.

Appropriate adhesion between the splint and the bandage improves the stability of the fracture fixation system. The peeling force should be at such a level that the gauze bandage is easy to peel with the bare hands and without causing unnecessary pain to the patient. A peel adhesion test was performed to determine the appropriate composition for the wood-plastic composite, featuring elevated adhesive forces when applied with various gauze bandages. Skin irritation tests were performed by Bioservice Scientific Laboratories GmbH, Planegg, Germany according to DIN EN ISO 1099310:2007 (ISO 10993-10:2002 + Amendment 1:2006) 'Test for irritation and delayed and delayed-type hypersensitivity'.

\section{Patient Study}

The suitability of the novel wood-plastic composite cast was evaluated in a patient series. Patients (26 females, 7 males, mean age 61 years) who had sustained a distal radius fracture were chosen for the study. The fractures were classified according to the AO classification as A2 $(n=19), B 1$ $(\mathrm{n}=1), \mathrm{C} 1(\mathrm{n}=9)$, and C2 (n=4) (AO Foundation, Davos, Switzerland). All patients were enrolled in a single hospital (Töölö Hospital, HUCH). Written informed consent was obtained from the study participants. The inclusion criteria were a distal radius fracture needing a cast, fracture not older than 14 days, age of the patient 17-90 y, mother tongue of the patient: Finnish or Swedish. The exclusion criteria were an open fracture, other fractures including those of the hand, a previous fracture of the distal radius or ulna, a previous or simultaneous tendon-, nerve- or vascular injury to the upper extremity, a multiply injured patient, decreased cooperation of the patient, malignancy, or an illness affecting the general health of the patient. Both conservatively treated patients, as well as operatively treated patients, needing casting were included.

All patients were treated according to the normal protocol of the hospital for treating distal radius fractures, including both conservative and operative treatment. The cast was applied by certified orthopedic technicians $(N=10)$ who were instructed in how to use the new composite cast. A single layer of a stocking was applied. A preheated composite cast was evaluated to estimate the cutting lines and final length, leaving the thumb and fingers free. After cutting, the cast was laid down on a single layer of cotton wool $(10 \mathrm{~cm}$ Soffban ${ }^{\circledR}$ Plus, BSN Medical GmbH, +Co. KG, Hamburg, Germany) that spontaneously attached to the cast. The cast was applied to the limb and wrapped in elastic bandages ( Soffcrepe $^{\circledR} 7.5$ × $3 \mathrm{~m}$, Crepe bandage B.S. Type 2, BSN Medical Ltd., Hull, East Yorkshire, England), using a mean of $1.6(\mathrm{n}=1$ or 2$)$ rolls and Mollelast ${ }^{\circledR}$ haft $8 \mathrm{~cm} \times 20 \mathrm{~m}$ (Lohmann \& Rauscher International GmbH \& Co. KGa, Rengsdorf, Germany). In 19 patients, ice packs were used during repositioning for cooling of the cast. Thereafter, the orthopedic technician responded to a questionnaire. Parameters concerning the use of the composite cast, status of the skin, and the opinion of the patient were recorded.

The follow-up was conducted according to the normal protocol of the hospital for conservatively or operatively treated distal radius fractures. During the study, the patients were treated or examined at the outpatient department by orthopedic surgeons or residents $(\mathrm{N}=17)$. At the time the composite cast was removed, the surgeons responded to a questionnaire. The following parameters were detected: immobilization time, conservative / operative treatment, rerepositioning, mechanical properties of the cast, skin appearance or irritation caused by the cast, allergic reactions, and subjective opinion of the patient. All parameters were collected in clinical report forms and transferred to a computerized database for detailed analyses. The clinical trial was monitored by an independent source (Clinius Ltd., Espoo, Finland), specialized in supporting healthcare companies in meeting regulatory requirements.

\section{RESULTS}

\section{The Wood-Plastic Material}

The cast is hard at room- and body temperature, but becomes freely moldable at temperatures over $+62{ }^{\circ} \mathrm{C}$. This can be achieved, using the automatic contact heater provided by the manufacturer. The cast retains moldable properties for 10-15 min but can also be stored in the heater, ready-to-use, for days. The temperature achieved is high enough to soften the plate, but due to the thermal characteristics of the wood and woody components, the cast does not feel hot to the skin, either on the operating personnel or on the patient. The cast can be handled without gloves and cut with conventional scissors into a desired shape without wrinkling or tearing of the edges. The material is nonsticky on the skin, but attaches to cottonwool layers and bandages. The cast remains applicable 5-10 min after heating and hardens during cooling (Fig. 1). The cooling process does not affect the shape of the molded cast. If rapid hardening is needed, the cast can be cooled with an ice pack. Reheating, reshaping, and repeated molding of the cast do not influence the material characteris- 


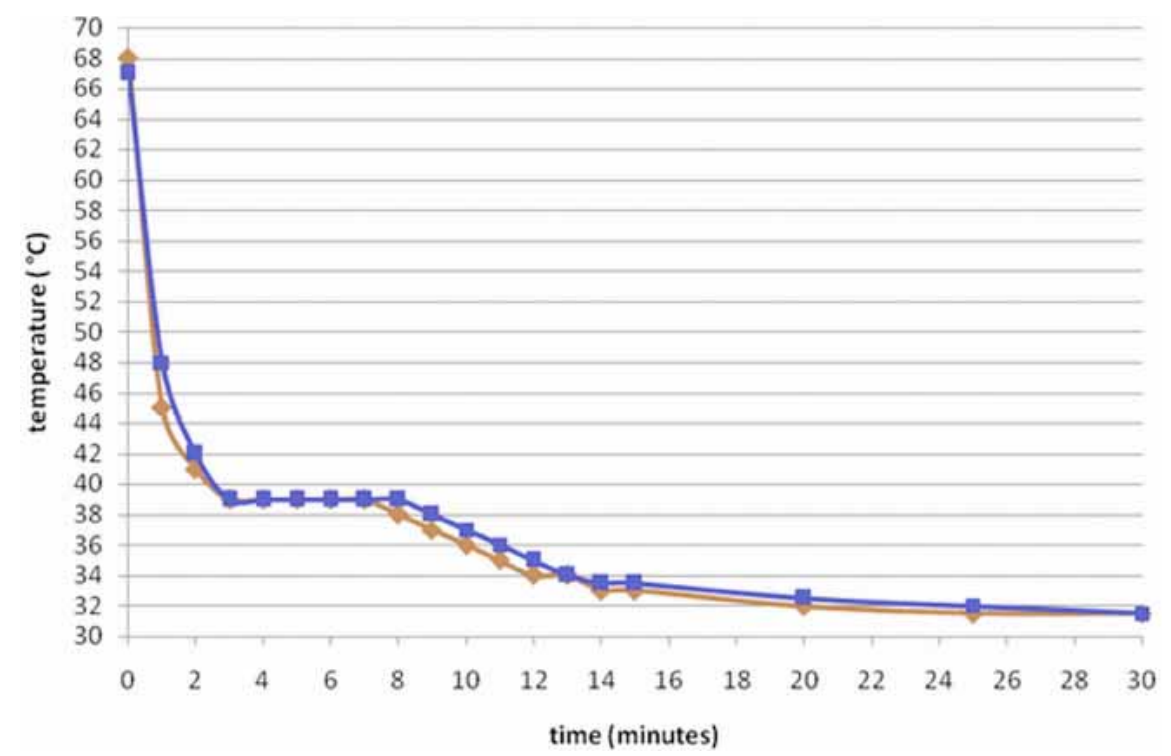

Fig. (1). Cooling behavior of the composite cast after positioning directly on the skin.

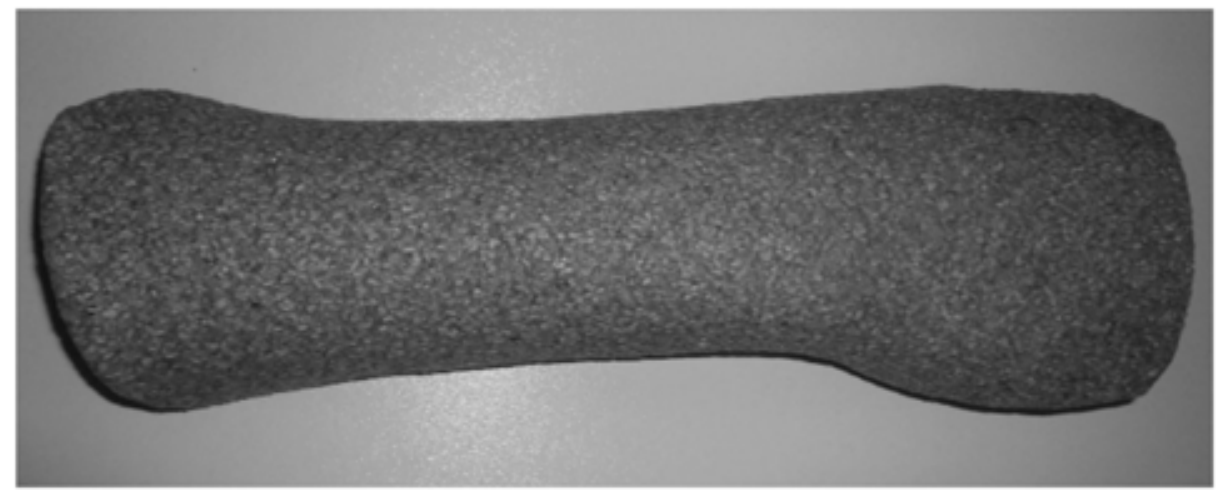

Fig. (2). A molded cast used for treatment of a distal radius fracture.

tics of the cast, resulting in a theoretically unlimited working time for the clinician.

\section{Mechanical Strength, Adhesion Properties, and Skin Irri- tation Test of the Wood-Composite Material}

The wood-plastic composite possesses strong selfadhesive properties under minor pressure. After the bond is formed, the segments cannot be separated from each other by the hands. Depending on the pressing force utilized, adhesion forces above $100 \mathrm{~N}$ were achieved, which in casting applications can be considered an 'everlasting' bond that cannot be broken without a casting saw. The stress at yield is $\sim 15 \mathrm{MPa}$ and the Young's modulus $500 \mathrm{MPa}$. The threepoint bending force of the wood-plastic composite was measured according to ISO 178.

Depending on the pressing force utilized, average peeling values between $1 \mathrm{~N}$ and $24 \mathrm{~N}$ were obtained. This bonding force level enables attachment of the bandage material to the cast, but can easily be peeled off afterwards. No irritation was observed and the final grade for the outcome of the study was negligible.

\section{Patient Study}

All 33 patients completed the trial. The mean time for applying the cast was $5.3(1.5-12.5)$ min (one patient ex- cluded due to a mistake in answering the questionnaire concerning the time). The orthopedic technicians stated that preheating of the cast occurred without problems in $32 / 33$, the cast was easy to mold in $31 / 33$, and easy to apply in the correct position in 30/33 of the cases. In one case, the cast hardened too quickly and in one, the technician did not answer the question. In 32/33 of the cases, the bandages were kept in favourable positions (one technician did not answer the question). In all, $31 / 33$ of the cases, the orthopedic technicians $(n=10)$ responded that they would favor future use of the composite cast if it were available; one technician was not certain after only a single use and one did not answer the question.

The average time for wearing the cast was 4.2 (1-6) weeks. Six patients were treated operatively with a volar plate, while the rest underwent repositioning of the wrist. The surgeon stated that the cast kept its mechanical properties and contour in 32/33 of the cases (one technician did not answer the question). A cast used for treatment of a distal radius fracture is shown in Fig. (2). The cast is hardly visible in X-rays (Fig. 3).

In 10 patients (six did not answer the question), the cast pressed into the skin, most often at the region of the distal ulna. In 10 cases, the skin was also minimally rubbed. No allergic reactions were observed. In two cases re- 


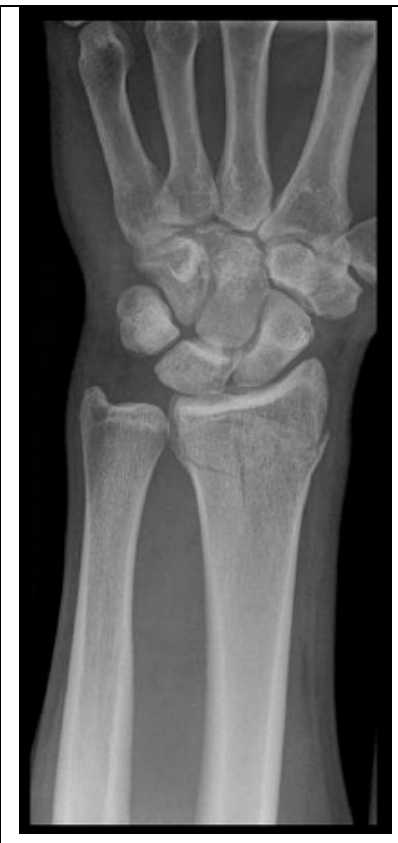

(3a)

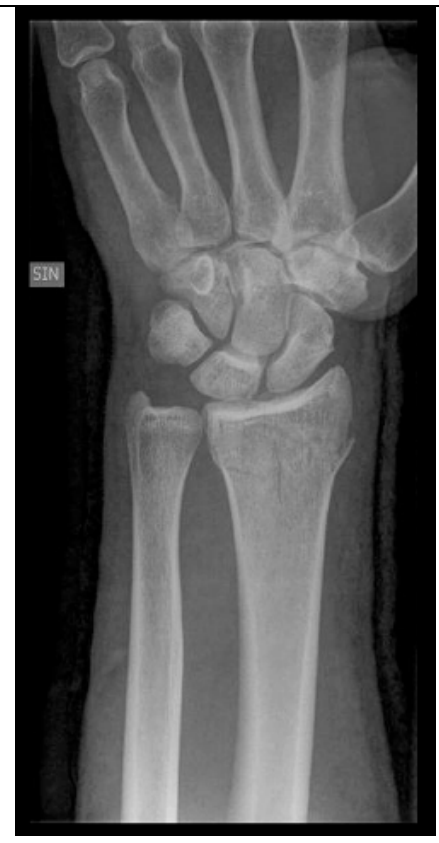

(3b)

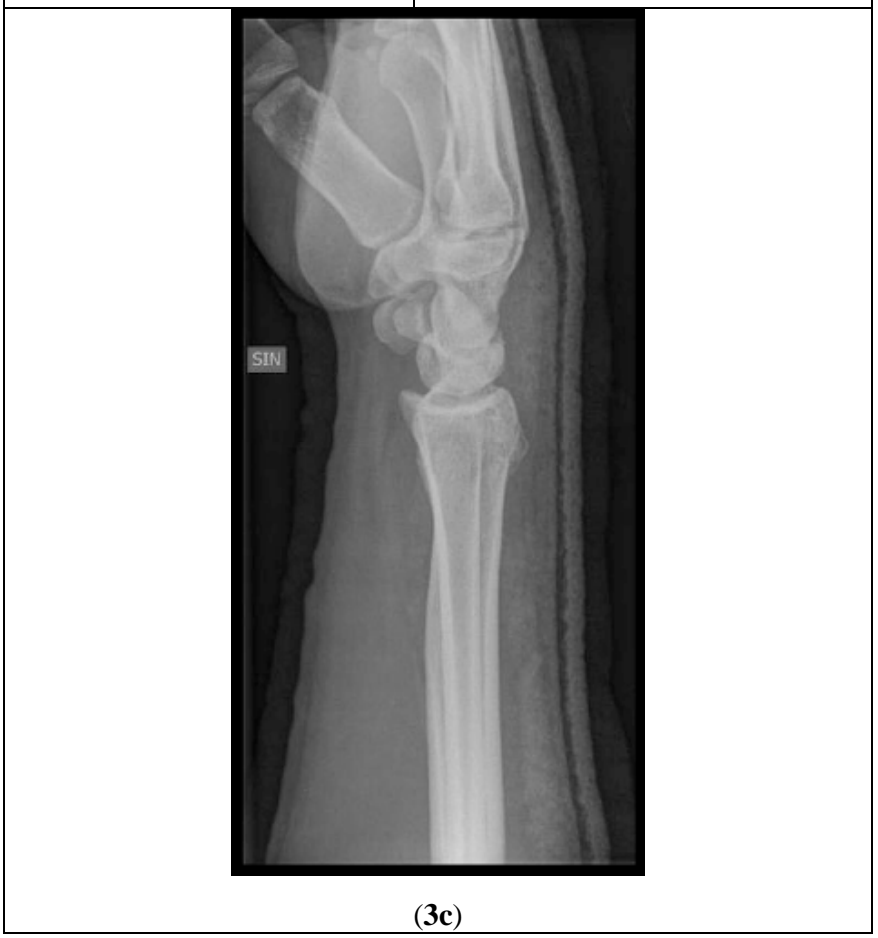

Fig. (3). Distal radius fracture a) before casting, b, c) after immobilization using the composite cast. Note the favorable moldable properties of the cast observed in $\mathbf{3 c}$ ).

repositioning was performed and a cast made of plaster of Paris was applied according to the study protocol. In one patient, the acceptable position of the distal radius was lost during the follow-up, but there was no evidence indicating that the situation was caused by the composite cast.

In all, 27 patients responded to the question on patient satisfaction. Patient satisfaction was high. Typical comments by the patients included: the cast feels nice when it is warm during application, the cast feels very light, and feels better

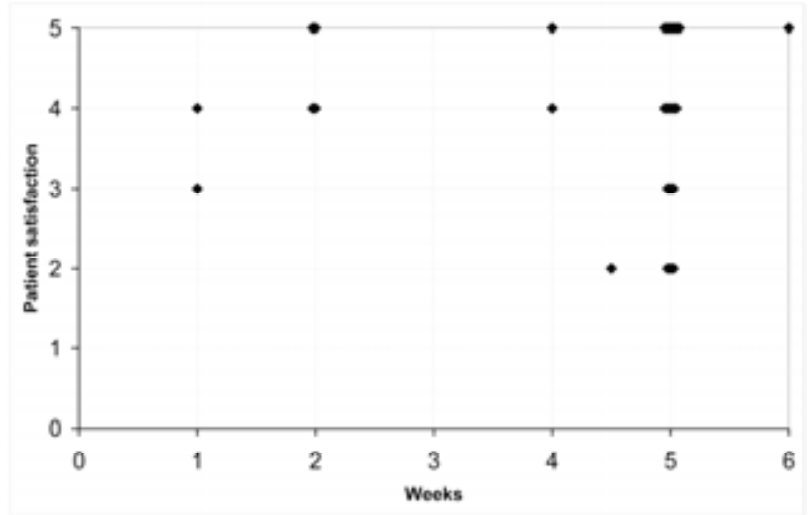

Fig. (4). Patient satisfaction and immobilization time.

than a cast made of plaster of Paris. The cast was considered to be very comfortable or comfortable by 18 of the patients, four had a neutral opinion, and five thought it was unpleasant. The main reason for the unpleasant feeling was too tight bandages or pressure of the composite cast on the skin. Patient satisfaction and immobilization time are shown in Fig. (4).

\section{DISCUSSION}

The novel nontoxic composite cast offers extreme rigidity in combination with favourable molding properties and a lightweight structure, thus eliminating several problems related to conventional splinting. Plaster of Paris casts are heavy and fragile, application of the cast is messy, and the drying time is long. It is not rare for the plaster of Paris cast to fracture during treatment, especially if it accidentally becomes wet. The major benefit of this traditional material is the absolutely free molding capability, especially in treating fractures where repositioning of bone fragments is needed. Fiberglass-reinforced polyurethane resin-based casting materials are considered practical alternatives for conventional plaster of Paris casts and are currently widely used in fracture treatment. These materials are lightweight, durable, waterproof, and tend to have shorter setting times than traditional plaster-based materials [10, 11]. However, fiberglass/polyurethane-based casting materials contain toxic or harmful components and, therefore, protective gloves must be used during application of the casting material. Protection of the patients' skin is also imperative. Both early- and latephase asthmatic reactions can be caused by occupational exposure to methylene diphenyl diisocyanate-containing casts, resulting in occupationally diagnosed illness [8]. These casts can also be considered as problem wastes, because they do not degrade in the environment. Activation of the hardening process of plaster of Paris and fiberglass/polyurethanebased casting materials are based on a water-initiated chemical reaction. Once the reaction has been initiated, the hardening process cannot be stopped or paused. A delay during application of the cast will lead to an unusable cast, which must then be replaced with a new one. The composite cast is remoldable and can even be reheated if the clinical practitioner needs to reformulate the shape of the cast. The manufacturer stated that the material itself can be heated up to +120 ${ }^{\circ} \mathrm{C}$ without disturbing the characteristics of the material. In clinical use, a temperature between $+64{ }^{\circ} \mathrm{C}$ and $+66{ }^{\circ} \mathrm{C}$ is recommended. 
The radiological properties of the composite cast appear to be excellent. In one case the radiologist even missed the cast on X-ray. Typically, the cast is visible outside the bone area on the radial or ulnar side in anteroposterior (AP) projections or on the dorsal side over lateral X-rays. These are areas in which X-ray beams are tangential to the cast and are absorbed over a longer distance. At the actual fracture site, even the trabeculae of the bone were clearly visible, with no interference by the cast itself. We assumed that in treatment, e.g. of scaphoideum fractures, X-ray images can reliably be evaluated without removing the cast.

In the patient study, the number of patients per doctor (n $=33 / 17)$ or per orthopedic technician (33/10) was low, offering only an initial experience with the new material. However, since Töölö Hospital / HUCH, with a referral area of 1.5 million inhabitants, manages all complicated and demanding injuries in adults, all healthcare professionals who participated in this study have wide experience in fracture treatment. The short experience with the novel material also simultaneously prevents single opinions from affecting the overall results.

Although wearing a cast is usually not associated with a pleasant feeling, the acceptance of the cast in clinical use was surprisingly high. Many of the patients were initially admitted from healthcare centers, and had already had a traditional primary cast applied. After being treated with a wood-composite cast, the lightweight and unitching properties of the material were the most usual comments by patients. Since the composite cast has a true 3D molding capability without wrinkle formation, the study design included the use of only a single layer of thin cotton wool padding under the cast. This amount of padding was sufficient for most patients; however, 10 patients reported an unpleasant feeling, and even local pain during the immobilization period, usually in the region of the distal ulna. Despite this imminent discomfort, no major skin damage was seen. This discomfort can easily be avoided, using thicker padding and additional local padding in prominent bony areas.

\section{CONCLUSION AND TRIAL REGISTRATION}

The purpose for developing this new type of composite cast was initially to eliminate or minimize some of the problems related to conventional casting systems: toxicity, lim- ited 3D molding, interference in X-rays, complexity of structures, or insufficient rigidity. Our results suggest that the composite cast is a potentially novel casting material that overcomes most of the issues with previous materials. It even offers some additional features, such as chemical bonding to itself, resulting in extremely strong bonds and junctions. It is also the first nontoxic, fully compostable casting material ever manufactured.

The patient study was registered at www.clinicaltrials.gov.

\section{CONFLICT OF INTEREST}

None declared.

\section{ACKNOWLEDEMENT}

None declared.

\section{REFERENCES}

[1] Re De Paepe JL, RaindorfGérard, C, Ed. Le Parlement Belge 18311894. Donnees biographiques. Brussels: Academie Royale de Belgique 1996

[2] Pirogov NI. Wikipedia, the free encyclopedia. Available from: http://en.wikipedia.org/wiki/Nikolay_Ivanovich_Pirogov.

[3] Salib P. Plaster casting. New York: Appleton-Century-Crofts 1975.

[4] Colditz J. Plaster of Paris: The forgotten hand splinting material. J Hand Ther 2002; 15:144-57.

[5] Bowler P, Powell ES. A clinical evaluation of plaster-of-Paris and eight synthetic fracture splinting materials. Injury 1992; 23:13-20.

[6] Cutler SW, Witmer D. Clinically evaluating the strength, durability and comfort of polyester casting tape as an alternative to fiberglass tape. J Nat Ass Orthop Tech Spring 2002:10-15.

[7] Redlich CA. Skin exposure and asthma. Is there a connection? Proc Am Thorac Soc 2010; 7:134-7.

[8] Donnelly R, Buick JB, Macmahon J. Occupational asthma after exposure to plaster casts containing methylene diphenyl diisocyanate. Occup Med 2004; 54:432-4.

[9] Suojalehto H, Linström I, Henriks-Eckerman M-L, Jungwelter S, Suuronen K. Occupational asthma related to low levels of airborne methylene diphenyl diisocyanate (MDI) in orthopedic casting work. Am J Ind Med 2011; 54:906-10.

[10] Herzig S, Muller J, Schuren J. A comparative study of the number of replacements required and application times for synthetic casts, combicasts and plaster-of-Paris. J Orthop Nurs 1999; 3:193-6.

[11] Marshall PD, Dible AK, Walters TH, Lewis D. When should a synthetic casting material be used in preference to plaster-of-Paris? A cost analysis and guidance for casting departments. Injury 1992; 23:542-4.

Received: September 26, $2011 \quad$ Revised: January 14, 2012

Accepted: January 17, 2012

(C) Lindfors and Salo; Licensee Bentham Open.

This is an open access article licensed under the terms of the Creative Commons Attribution Non-Commercial License (http://creativecommons.org/licenses/by-nc/3.0/) which permits unrestricted, non-commercial use, distribution and reproduction in any medium, provided the work is properly cited. 